\title{
Risk Return Relationship in the Portfolio Selection Models
}

\author{
Ken Hung', C. W. Yang'2, Yifan Zhao' ${ }^{2}$, Kuo-Hao Lee ${ }^{3 *}$ \\ ${ }^{1}$ Texas A \& M International University, Laredo, TX, USA \\ ${ }^{2}$ Clarion University of Pennsylvania, Clarion, PA, USA \\ ${ }^{3}$ Department of Finance, Zeigler College of Business, Bloomsburg University of Pennsylvania, Bloomsburg, PA, USA \\ Email: *klee@bloomu.edu
}

How to cite this paper: Hung, K., Yang, C.W., Zhao, Y.F. and Lee, K.-H. (2018) Risk Return Relationship in the Portfolio Selection Models. Theoretical Economics Letters, 8, 358-366.

https://doi.org/10.4236/tel.2018.83025

Received: October 12, 2017

Accepted: February 9, 2018

Published: February 12, 2018

Copyright $\odot 2018$ by authors and Scientific Research Publishing Inc. This work is licensed under the Creative Commons Attribution International License (CC BY 4.0).

http://creativecommons.org/licenses/by/4.0/

\begin{abstract}
In this paper, we calculate four different kinds of means-AM, GM, HM, and GDM-to investigate the risk-return contour using Markowitz risk minimization and Sharpe's angle maximization models. For a given $k$ value (target portfolio return), the rank order of risk or variance-covariance $(v)$ can change. In the vertical segment of an efficient frontier curve, we observed $v(\mathrm{GDM})>$ $\mathrm{v}(\mathrm{HM})>\mathrm{v}(\mathrm{GM})>\mathrm{v}(\mathrm{AM})$. At higher $k$ values, the rank changes to $\mathrm{v}(\mathrm{GDM})>\mathrm{v}(\mathrm{HM})>\mathrm{v}(\mathrm{AM})>\mathrm{v}(\mathrm{GM})$. That is to say, ranking a portfolio using different kinds of means may well give different rankings depending on what $k$ value one is evaluating. It is also shown the harmonic mean should not be used in the case of a small negative growth rate in stock prices.
\end{abstract}

\section{Keywords}

Arithmetic Mean, Geometric Mean, Golden Mean, Harmonic Mean, Markowitz Risk Minimization, Sharpe's Angle Maximization

\section{Introduction and Literature Reviews}

The foundation of modern investment theory is laid upon the quadratic program portfolio selection model developed more than half century ago by Harry Markowitz [1] [2] [3]. The optimization (risk-minimization) process over meanvariance-covariance space can trace out the efficient frontier curve, which provides the solution space for investors. However, an exact solution cannot be found without the knowledge of a risk free rate on a government bond and an investor's attitude toward risk. To this end, Sharpe [4] formulated and solved the angle-maximization model in which the risk (standard deviation) adjusted port- 
folio return (net of risk free rate) was maximized. The Sharpe model provides a convex combination of risk free government bonds and a portfolio of stocks selected based on the criterion of risk minimization. Attitude toward risk such as $20 \%$ on bond and $80 \%$ on stocks will give investor an exact solution without the knowledge of the indifference (isoutility) curve. Soto and $\mathrm{Su}$ [5] proposed a "sparse" estimator of the inverse covariance matrix that achieves significant outof-sample risk reduction and improves certainty equivalent returns after transaction costs. Yang et al. [6] proved that Markowitz risk minimization and Sharpe angle-maximization models are mathematically equivalent given some required portfolio returns and risk free bond rate. Gil-Bazo [7] found the return dynamics is related to the investor's portfolio choice for different investment horizons and that return predictability under stationarity which may induce both positive and negative horizon effects in the optimal allocation to the risky asset. Best and Grauer [8] investigated and revealed when only a budget constraint is imposed on the investment problem, the analytical results indicate that an MV-efficient portfolio's weights, mean, and variance can be extremely sensitive to changes in asset means.

Does the choice of mean returns of stocks in the portfolio matter in the selection process? If so, how different are the optimum solution sets? In this note, we first apply the well-known means: arithmetic, geometric and harmonic means to five companies stocks. In addition, we add a golden mean to the simulation for comparison. The organization of the paper is as follows. Next section introduces the Markowitz risk minimization and Sharpe angle maximization models. Section III describes data and four different means. Section IV performs computer simulations via LINGO [9] to trace out corresponding efficient frontier curves. Section $\mathrm{V}$ gives a conclusion.

\section{Portfolio Selection Models with Different Means}

Given a set of $\mathrm{n}$ selectable stocks, the purpose of the Markowitz portfolio model is to minimize the weighted risk in terms of variance and covariance of $n$ stock returns, or

$$
\begin{gathered}
\text { Minimize } v=\sum_{i} x_{i}^{2} \sigma_{i}^{2}+\sum_{i} \sum_{i \neq j} x_{i} x_{j} \sigma_{i j} \\
\text { Subject to } \sum_{i} x_{i} \overline{R_{i}} \geq k \\
\sum_{i} x_{i}=1 \\
x_{i} \geq 0
\end{gathered}
$$

where $x_{i}=$ the weight or proportion of investment in stock $i$;

$\sigma_{i}^{2}=$ variance of returns in stock $i$;

$\sigma_{i j}=$ covariance of return between stock $i$ and $j$;

$\overrightarrow{R_{i}}=$ expected or average rate of return of stock $i$;

$k=$ target portfolio rate of return. 
Note that rate of return is frequently calculated as $\frac{P_{t}-P_{t-1}}{P_{t-1}}$ on which mean, variance and covariance are calculated. Optimum weights $\left(x_{1}^{*}, x_{2}^{*}, \cdots, x_{n}^{*}\right)$ for $x_{i}^{*} \geq 0$ are the framework under which weighted risk $v$ can be calculated. Along with a set of $k$ values, we have geometric means calculated according to the growth rate formula (next section) and a set of risk-return values on which an efficient frontier curve can be traced.

However, the exact location cannot be determined with a risk-free bond rate $R_{F}$. To expand to risk minimization model, Sharpe [4] proposed an anglemaximizing model with a highest straight line from $R_{F}$ that is tangent to the efficient frontier derived from the Markowitz model.

$$
\begin{gathered}
\text { Maximize } \tan \theta=\frac{\sum_{i} x_{i}\left(\overline{R_{i}}-R_{F}\right)}{\left(\sum_{i} x_{i}^{2} \sigma_{i}^{2}+\sum_{i} \sum_{j} x_{i} x_{j} \sigma_{i j}\right)^{1 / 2}} \\
\text { Subject to } \sum_{i} x_{i}=1 \\
x_{i} \geq 0
\end{gathered}
$$

As is shown by Yang et al. [6], a given $R_{F}$ corresponds to a $k$ value in equation (2) of the Markowitz model. Furthermore, the denomination of Equation (5) is the square root of the objective function of Equation (1). Thus, the Markowitz and Sharpe models exhibit reciprocal relations for a given set of $\overline{R_{i}}, R_{F}$ and $k$ value.

\section{Description of Data and Characteristics of Different Mean Returns}

Monthly stock price of 5 companies, Mastercard Incorporated (MA), International Business Machines Corp. (IBM), Johnson \& Johnson (JNJ), McDonald's Corp. (MCD), Wal-Mart Stores Inc. (WMT), from September of 2007 to August of 2008 are calculated to obtain $55\left(11^{\star} 5\right)$ rates of return [10]. The arithmetic means (AM) and associated variance and covariance of stock returns are reported in Table 1.

An examination on five arithmetic means indicates the highest return is by Masters Charge (MA), followed by Wal-Mart (WMT), McDonalds (MCD), Johnson \& Johnson (JNJ) and International Business Machine (IBM). As an alternative to arithmetic mean is the often used geometric mean:

$\left(\frac{P_{2}}{P_{1}} \cdot \frac{P_{3}}{P_{2}} \cdot \frac{P_{4}}{P_{3}} \cdots \frac{P_{n}}{P_{n-1}}\right)^{\frac{1}{n}}-1=\left(\frac{P_{n}}{P_{1}}\right)^{\frac{1}{n}}-1$. Note that when $\frac{P_{i+1}}{P_{i}}$ exceeds (falls short of) one it implies a position (negative) growth rate as is measured by $\frac{P_{i+1}}{P_{i}}-1=\frac{P_{i+1}-P_{i}}{P_{i}}$. Viewed in this light, an arithmetic mean (AM) is derived additively whereas its geometric mean (GM) is calculated multiplicatively using 
Table 1. Means (\%) and variances (\%) of five stock returns.

\begin{tabular}{cccccc}
\hline & $\mathrm{MA}$ & $\mathrm{IBM}$ & $\mathrm{JNJ}$ & $\mathrm{MCD}$ & WMT \\
\hline $\mathrm{AM}$ & $5.4499 \%$ & $0.6093 \%$ & $0.9410 \%$ & $1.7303 \%$ & $3.0283 \%$ \\
$\mathrm{GM}$ & $4.6235 \%$ & $0.4385 \%$ & $0.8756 \%$ & $1.6012 \%$ & $2.9526 \%$ \\
$\mathrm{HM}$ & $-6.9923 \%$ & $-28.3500 \%$ & $3.1584 \%$ & $4.2577 \%$ & $0.1983 \%$ \\
$\mathrm{GDM}$ & $12.2563 \%$ & $1.4511 \%$ & $1.9342 \%$ & $2.5252 \%$ & $5.1867 \%$ \\
$\mathrm{REG}$ & $4.5127 \%$ & $1.3001 \%$ & $0.6101 \%$ & $0.9135 \%$ & $3.012 \%$ \\
$\sigma_{A M}^{2}$ & $1.7700 \%$ & $0.3386 \%$ & $0.1316 \%$ & $0.2573 \%$ & $0.1562 \%$ \\
$\sigma_{G M}^{2}$ & $1.7768 \%$ & $0.3388 \%$ & $0.1317 \%$ & $0.2575 \%$ & $0.1562 \%$ \\
$\sigma_{H M}^{2}$ & $3.3181 \%$ & $8.7250 \%$ & $0.1808 \%$ & $0.3212 \%$ & $0.2362 \%$ \\
$\sigma_{G D M}^{2}$ & $2.2333 \%$ & $0.3456 \%$ & $0.1415 \%$ & $0.2636 \%$ & $0.2027 \%$ \\
$\sigma_{R E G}^{2}$ & $1.7790 \%$ & $0.3433 \%$ & $0.1328 \%$ & $0.2642 \%$ & $0.1562 \%$ \\
\hline
\end{tabular}

$\mathrm{AM}=$ arithmetic mean; $\mathrm{GM}=$ geometric mean; $\mathrm{HM}=$ harmonic mean; $\mathrm{GDM}=$ golden mean; $\mathrm{REG}=$ regression-based mean; $\sigma_{A M}^{2}=$ variance of stock returns using AM as the central location; MA = Mastercard Incorporated; IBM = International Business Machines Corp; JNJ = Johnson \& Johnson; MCD = McDonald's Corp.; WMT = Wal-Mart Stores Inc.

the same measure of rate of return, $\frac{P_{i+1}}{P_{i}}-1$. Well-known in statistics, AM is more sensitive to outliers than is GM and as such GM may be preferred in such cases. During the sample period, the five stock prices underwent substantial changes. For example, the greatest monthly price change was $28.4 \%$ while the largest drop registered $13.97 \%$ for Masters Charge. From the perspective of risk averseness, AM might not be preferred. Calculated geometric mean returns follow the same rank order as that of arithmetic mean returns (Table 1).

A harmonic mean may be appropriate for a variable that measures rates of change (e.g., velocity). In business applications, number of shares of stocks of a national fund, a unit of price (e.g., $\$ 1,000,000$ ) can purchase could fit into this category. The harmonic mean (HM) is calculated as $\frac{1}{\mathrm{HM}}=\frac{\sum_{i=1}^{n} \frac{1}{x_{i}}}{n}$ where $x_{i}$ represents rate of return for stock $i$. For $n=2$ and $x_{i}>0$, the geometric mean is the square root of arithmetic and harmonic means or $\mathrm{GM}^{2}=\mathrm{AM} \cdot \mathrm{HM}$. For a set of clustered numbers, the three means produce very similar values. However, $\mathrm{HM}$ can be very biased toward a negative value in the presence of a small negative return, i.e., $x_{i}=-0.02$ implies $\frac{1}{x_{i}}=-50$ which will dominate other positive regular returns. This is the case we encounter in calculating HM for IBM and MA (e.g., $x_{11}=-0.66 \%$ for MA and $x_{4}=-0.91 \%$ for IBM). For comparison, we report the HM return in Table 1 as well.

The golden mean, also known as golden section and golden ratio from Leonardo da Vinci is a special case of the geometric mean on line segment $|A C|=1$ : 
A location $B$ between points $A$ and $C$ such as $\frac{|A C|}{|A B|}=\frac{|A B|}{|B C|}$ which leads to the solution $\frac{-1+\sqrt{5}}{2}=0.618034$. In another word, it is the range $\left(x_{\max }-x_{\min }\right)$ that determines the value of golden mean (GDM) since it is hinged calculated as $x_{\min }+0.618034 *\left(x_{\max }-x_{\min }\right)$. For instance, the GDM return for MA is $-13.97 \%+0.618034 *(28.47 \%-(-13.97 \%))=12.256 \%$. When the range plays a key role in determining a representative value, GDM may be a viable candidate and are reported in Table 1. The corresponding variances and covariances of stock returns using the AM, GM, HM, and GDM are shown in Table 2.

Table 2. Variance and covariance using five means.

\begin{tabular}{|c|c|c|c|c|c|}
\hline & $M A$ & $I B M$ & $J N J$ & $M C D$ & $W M T$ \\
\hline \multicolumn{6}{|c|}{$\underline{\text { Arithmetic mean }}$} \\
\hline$M A$ & 0.0177 & & & & \\
\hline$I B M$ & 0.001091 & 0.003386 & & & \\
\hline$J N J$ & 0.001188 & 0.000313 & 0.001316 & & \\
\hline$M C D$ & 0.003895 & 0.000866 & 0.001199 & 0.002573 & \\
\hline$W M T$ & 0.002857 & $5.42 \mathrm{E}-05$ & 0.000616 & 0.000442 & 0.001562 \\
\hline \multicolumn{6}{|c|}{$\underline{\text { Geometric mean }}$} \\
\hline$M A$ & 0.017768 & & & & \\
\hline$I B M$ & 0.001105 & 0.003388 & & & \\
\hline$J N J$ & 0.001193 & 0.000314 & 0.001317 & & \\
\hline$M C D$ & 0.003906 & 0.000868 & 0.0012 & 0.002575 & \\
\hline$W M T$ & 0.002863 & $5.55 \mathrm{E}-05$ & 0.000616 & 0.000443 & 0.001562 \\
\hline \multicolumn{6}{|c|}{$\underline{\text { Harmonic mean }}$} \\
\hline$M A$ & 0.033181 & & & & \\
\hline$I B M$ & 0.037122 & 0.08725 & & & \\
\hline$J N J$ & -0.00157 & -0.00611 & 0.001808 & & \\
\hline$M C D$ & 0.000751 & -0.00645 & 0.001759 & 0.003212 & \\
\hline$W M T$ & 0.006378 & 0.00825 & $-1.20 \mathrm{E}-05$ & -0.00027 & 0.002362 \\
\hline \multicolumn{6}{|c|}{$\underline{\text { Golden mean }}$} \\
\hline$M A$ & 0.022333 & & & & \\
\hline$I B M$ & 0.001664 & 0.003456 & & & \\
\hline$J N J$ & 0.001864 & 0.000397 & 0.001415 & & \\
\hline$M C D$ & 0.004436 & 0.000933 & 0.001278 & 0.002636 & \\
\hline$W M T$ & 0.004326 & 0.000236 & 0.00083 & 0.000614 & 0.002027 \\
\hline \multicolumn{6}{|c|}{$\underline{\text { Regression-based mean }}$} \\
\hline$M A$ & 0.01779 & & & & \\
\hline$I B M$ & 0.001025 & 0.003433 & & & \\
\hline$J N J$ & 0.00122 & 0.00029 & 0.001328 & & \\
\hline$M C D$ & 0.003974 & 0.000808 & 0.001227 & 0.002642 & \\
\hline$W M T$ & 0.00286 & 0.000052 & 0.000617 & 0.000444 & 0.001562 \\
\hline
\end{tabular}




\section{A Comparison of Simulation Results}

An efficient frontier generally consists of two parts: a vertical section and a concave part. The vertical part indicates Equation (2) holds with strict inequality (> $k$ ). That is the portfolio return at optimality exceeds the minimum required rate $k$. For $k=2 \%$ (annualized), which is obviously too low. The optimum portfolio return far exceeds $k=2 \%$ in the case of AM. If we arbitrarily impose Equation (2) with an equality sign $(k=2 \%)$, there will exist no feasible solution, for the lowest annual average rate of return is $7.317 \%$ (IBM), and as such the vertical sections of the efficient frontier curve starts to bend at $k \approx 20 \%, k=19 \%, k=$ $18 \%$, and $k=31 \%$ in the cases of AM, GM, HM and GDM respectively (Table 3). A perusal of Table 3 indicates that for range of $k<18 \%$, the portfolio risks in the Markowitz model manifest the following rank order $0.1225(\mathrm{AM})<0.1226$ $(\mathrm{GM})<0.1421(\mathrm{HM})<0.1481$ (GDM) for each given $\mathrm{k}$ value.

As far as minimum risk level where efficient frontier is vertical is concerned, the risk levels of GM and HM are bounded by that AM curve and GDM. Given that GDM are highest in all four means, its variance-covariance is greatest at lower level of $k$ values. The variances of HM are even greater because of negative mean returns. However, five of ten covariances in HM are negative and thus the portfolio risk could be reduced from proper diversification. The high values of the GDM returns translate into higher variances and covariances when compared to that of AM and GM. It is little wonder that the efficient frontier has the largest risk in the vertical segment or $\mathrm{v}(\mathrm{GDM})>\mathrm{v}(\mathrm{HM})>\mathrm{v}(\mathrm{GM})>\mathrm{v}(\mathrm{AM})$. As $k$ value increases to $30 \%$ the risk become the greatest for HM that has 5 largest variances of all the four means. The rank of the portfolio risk become $\mathrm{v}(\mathrm{HM})>$ $\mathrm{v}(\mathrm{GM})>\mathrm{v}(\mathrm{AM})>\mathrm{v}(\mathrm{GDM})$. Note that $\mathrm{v}(\mathrm{GDM})$ is still at its vertical segment at

Table 3. Risk-return combination using five means.

\begin{tabular}{ccccccccccc}
\hline$k$ & $\mathrm{v}(\mathrm{AM})$ & rank & $\underline{\mathrm{v}(\mathrm{GM})}$ & rank & $\underline{\mathrm{v}(\mathrm{HM})}$ & rank & $\underline{\mathrm{v}(\mathrm{GDM})}$ & rank & $\underline{\mathrm{v}(\mathrm{REG})}$ & rank \\
\hline 0.02 & 0.122 & $<1>$ & 0.123 & $<3>$ & 0.142 & $<4>$ & 0.148 & $<5>$ & 0.122 & $<2>$ \\
0.06 & 0.122 & $<1>$ & 0.123 & $<3>$ & 0.142 & $<4>$ & 0.148 & $<5>$ & 0.122 & $<2>$ \\
0.1 & 0.122 & $<1>$ & 0.123 & $<3>$ & 0.142 & $<4>$ & 0.148 & $<5>$ & 0.122 & $<2>$ \\
0.14 & 0.122 & $<1>$ & 0.123 & $<3>$ & 0.142 & $<4>$ & 0.148 & $<5>$ & 0.122 & $<2>$ \\
0.18 & 0.122 & $<1>$ & 0.123 & $<3>$ & 0.142 & $<4>$ & 0.148 & $<5>$ & 0.122 & $<2>$ \\
0.22 & 0.124 & $<1>$ & 0.126 & $<3>$ & 0.143 & $<4>$ & 0.148 & $<5>$ & 0.124 & $<2>$ \\
0.26 & 0.133 & $<1>$ & 0.138 & $<3>$ & 0.147 & $<4>$ & 0.148 & $<5>$ & 0.134 & $<2>$ \\
0.3 & 0.152 & $<2>$ & 0.159 & $<4>$ & 0.16 & $<5>$ & 0.148 & $<3>$ & 0.153 & $<2>$ \\
0.34 & 0.187 & $<3>$ & 0.2 & $<5>$ & 0.185 & $<2>$ & 0.148 & $<1>$ & 0.189 & $<4>$ \\
0.38 & 0.253 & $<3>$ & 0.305 & $<5>$ & 0.222 & $<2>$ & 0.152 & $<1>$ & 0.291 & $<4>$ \\
0.42 & 0.372 & $<3>$ & 0.558 & $<4>$ & 0.271 & $<2>$ & 0.16 & $<1>$ & 0.568 & $<5>$ \\
0.46 & 0.565 & $<3>$ & 0.967 & $<4>$ & 0.333 & $<2>$ & 0.173 & $<1>$ & 1.038 & $<5>$ \\
0.5 & 0.831 & $<3>$ & 1.531 & $<4>$ & 0.429 & $<2>$ & 0.191 & $<1>$ & 1.703 & $<5>$ \\
\hline
\end{tabular}


$k=0.30 \%$ (see Table 3 ).

From $k \geq 0.34$ and on the risk in the case of GM become the greatest because the choice set has dwindled: only WMT (35.436\%) and MA (55.477\%) have the mean return greater than $34 \%$ and as such most of the weights go to WMT and MA regardless of their risk levels. On the contrary risk of GDM assumes its smallest value since most of the five stocks have higher returns: $147 \%$, $22.24 \%, 30.3 \%, 23.208 \%$ and $17.412 \%$ for MA, WMT, MCD, JNJ and IBM respectively. HM has shown smallest risk due to negative covariance. However, it must be pointed out that small negative returns are biased and misleading in the sense that the double reciprocity formula assigns too much weight to small negative return (slight price decrease in stock). The risk associated with AM for $k \geq 34 \%$ is slightly less than that of GM since GM is less sensitive to outliers than is AM, and as such AM has a slightly larger choice set (smaller risk) at high $k$ value. The four efficient frontiers are shown in Figure 1.

For a given risk free rate $R_{F}$, the greatest tangent angle attainable between the ray from $R_{F}$ and the efficient frontier curve can be calculated from Equation (5). By verifying $R_{F}$ we find the optimal tangent angle via LINGO and report the portfolio returns portfolio risk $(v)$ and tangent angles $(\tan \theta)$ in Table 4. An inspection of Table 4 reveals that Sharpe's solutions with GDM dominates that with $\mathrm{HM}$ for $R_{F}=3 \%, 4 \%, 5 \%$ and $6 \%$ because return under GDM are greater but risks smaller than that under HM. For $R_{F}=7 \%$ and $10 \%$, returns and risks are both greater under GDM in comparison to HM. Hence we calculate Sharpe's index orits $\tan \theta$ and used as the selection citation.

A comparison between AM and GM indicates that risk-return combinations using AM dominate that using GM: higher return with less risk from $R_{F}=3 \%$ through $7 \%$. At $R_{F}=10 \%$, both the portfolio return and risk are greater using AM: $0.3612>0.3465$ and $0.2184>0.2104$. As a consequence, we compare $\tan \theta$

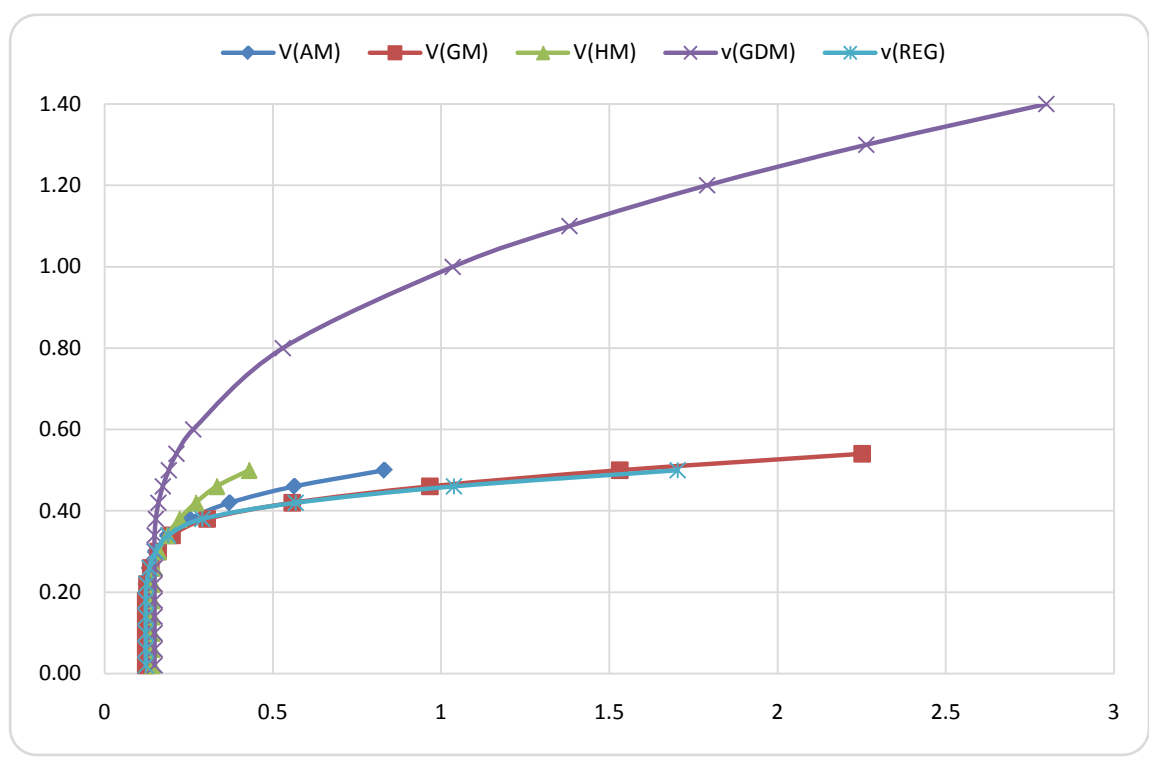

Figure 1. Efficient frontier curves using five different means. 
Table 4. Angle maximization solutions using five means.

\begin{tabular}{|c|c|c|c|c|c|c|}
\hline $\mathrm{RF}$ & Return \& Risk & $\mathrm{AM}$ & GM & $\mathrm{HM}$ & GDM & REG \\
\hline \multirow[t]{3}{*}{$\mathrm{RF}=3 \%$} & $\mathrm{Rp}$ & 0.3403 & 0.3339 & 0.4253 & 0.5939 & 0.3321 \\
\hline & $\mathrm{v}$ & 0.1874 & 0.1918 & 0.2782 & 0.2578 & 0.1787 \\
\hline & $\tan \theta$ & 0.7169 & 0.6939 & 0.7494 & 1.1106 & 0.7146 \\
\hline \multirow[t]{3}{*}{$\mathrm{RF}=4 \%$} & $\mathrm{Rp}$ & 0.3422 & 0.3353 & 0.4349 & 0.6073 & 0.3334 \\
\hline & $\mathrm{v}$ & 0.1898 & 0.1936 & 0.2922 & 0.2704 & 0.1804 \\
\hline & $\tan \theta$ & 0.6938 & 0.6712 & 0.7307 & 1.0911 & 0.6910 \\
\hline \multirow[t]{3}{*}{$\mathrm{RF}=5 \%$} & $\mathrm{Rp}$ & 0.3435 & 0.3368 & 0.4457 & 0.6219 & 0.3349 \\
\hline & $\mathrm{v}$ & 0.1914 & 0.1956 & 0.3085 & 0.2846 & 0.1822 \\
\hline & $\tan \theta$ & 0.671 & 0.6485 & 0.7124 & 1.0721 & 0.6675 \\
\hline \multirow[t]{3}{*}{$\mathrm{RF}=6 \%$} & $\mathrm{Rp}$ & 0.3449 & 0.3384 & 0.4481 & 0.6379 & 0.3365 \\
\hline & $\mathrm{v}$ & 0.1932 & 0.1978 & 0.3123 & 0.3008 & 0.1843 \\
\hline & $\tan \theta$ & 0.6482 & 0.6259 & 0.6945 & 1.0536 & 0.6441 \\
\hline \multirow[t]{3}{*}{$\mathrm{RF}=7 \%$} & $\mathrm{Rp}$ & 0.3464 & 0.3402 & 0.4501 & 0.6554 & 0.3383 \\
\hline & $\mathrm{v}$ & 0.1952 & 0.2004 & 0.3157 & 0.3195 & 0.1867 \\
\hline & $\tan \theta$ & 0.6255 & 0.6035 & 0.6766 & 1.0356 & 0.6209 \\
\hline \multirow[t]{3}{*}{$\mathrm{RF}=10 \%$} & $\mathrm{Rp}$ & 0.3612 & 0.3465 & 0.4572 & 0.6928 & 0.3445 \\
\hline & $\mathrm{v}$ & 0.2184 & 0.2104 & 0.328 & 0.3625 & 0.1961 \\
\hline & $\tan \theta$ & 0.5589 & 0.5373 & 0.6237 & 0.9846 & 0.5522 \\
\hline
\end{tabular}

$R p=\sum x_{i} \bar{R}_{i}=$ Portfolio return. $v=$ Variance and covariance of 5 stocks returns. $\tan \theta=\sum x_{i}\left(\bar{R}-R_{F}\right) / \sqrt{v}=$ Portfolio premium net of risk free rate per risk.

and find that under AM is greater $0.5589>0.5373$.

\section{Concluding Remarks}

In this paper, we calculate four different kinds of means-AM, GM, HM, and GDM - to investigate the risk-return contour using Markowitz risk minimization and Sharpe's angle-maximization models. For a given $k$ value (target portfolio return), the rank order of risk or variance-covariance $(v)$ can change. In the vertical segment of an efficient frontier curve, we observed $\mathrm{v}(\mathrm{GDM})>\mathrm{v}(\mathrm{HM})>$ $\mathrm{v}(\mathrm{GM})>\mathrm{v}(\mathrm{AM})$. At higher $k$ values, the rank changes to $\mathrm{v}(\mathrm{GDM})>\mathrm{v}(\mathrm{HM})>$ $\mathrm{v}(\mathrm{AM})>\mathrm{v}(\mathrm{GM})$. That is to say, ranking a portfolio using different kinds of means may well give different rankings depending on what $k$ value one is evaluating.

When a risk free-rate is added to the Markowitz model, we arrive at the angle-maximization solution. It seems that risk-return combinations under GDM dominate those under HM for the former has high returns and less risk. The same can be said of AM and GM. The combination using AM seems to have greater portfolio returns but less risk. When does these exists a trade-off, i.e., higher risk coupled with higher return, Sharpe's index $(\tan \theta)$ favors both GDM 
and $\mathrm{AM}$ over $\mathrm{HM}$ and GM respectively for greater angle translates into high portfolio return (net of $R_{f}$ ) per risk. Care must be exercised though; the results from this paper are limited to the stocks that we take in the sample. However, comparative evaluations are needed for a comprehensive analysis on a portfolio performance. In sum, GM is less sensitive to outliers and hence is more suitable for conservative strategy. GDM is determined by the size of its range and tends to offer an optimistic forecast on mean return especially when there exist a few unusually large positive returns. Finally, HM is not appropriate if some of the returns (\%) are small and negative. In that case, HM ought to be removed from the analysis.

\section{References}

[1] Markowitz, H. (1952) Portfolio Selection. Journal of Finance, 7, 77-91.

[2] Markowitz, H. (1952) The Optimization of a Quadratic Function Subject to Linear Constraints. Naval Research Logistics Quarterly, 3, 111-133. https://doi.org/10.1002/nav.3800030110

[3] Markowitz, H. (1959) Portfolio Selection: Efficient Diversification of Investment. John Wiley \& Sons, Inc., New York.

[4] Sharpe, W.F. (1964) Capital Asset Prices: A Theory of Market Equilibrium under Condition of Risk. Journal of Finance, 19, 425-442.

[5] Goto, S. and Xu, Y. (2015) Improving Mean Variance Optimization through Sparse Hedging Restrictions. Journal of Financial \& Quantitative Analysis, 50, 1415-1441. https://doi.org/10.1017/S0022109015000526

[6] Yang, C.W., Hung, K. and Yang, F.A. (2002) A Note on the Markowitz Risk Minimization and the Sharpe Angle Maximization Models. In: Lee, C.F., Ed., Advances in Investment Analysis and Portfolio Management, Vol. 9, Elsevier Science, Amsterdam.

[7] Gil-Bazo, J. (2006) Investment Horizon Effects. Journal of Business Finance \& Accounting, 33, 179-202. https://doi.org/10.1111/j.1468-5957.2006.01098.x

[8] Best, M.J. and Grauer, R.R. (1991) On the Sensitivity of Mean-Variance-Efficient Portfolios to Changes in Asset Means: Some Analytical and Computational Results. The Review of Financial Studies, 4, 315-342. https://doi.org/10.1093/rfs/4.2.315

[9] LINGO 8.0 Linear and Nonlinear Optimizer. LINGO System Inc., Chicago, IL, 2003.

[10] Elton, E.J., Gruber, M.J., Brown, S.J. and Goetzmann (2007) Modern Portfolio Theory and Investment Analysis. $7^{\text {th }}$ Edition, John Wiley and Sons, Inc., Hoboken, NJ. 